\title{
Gastric Antral Webs: Anatomical Variants and Appropriate Endotherapy
}

\author{
Hema V. Varadarajulu ${ }^{1}$ Kavitha Mohanasundaram² \\ ${ }^{1}$ Department of Medical Gastroenterology, Sree Balaji Medical \\ College and Hospital, Chennai, Tamil Nadu, India \\ 2Department of Rheumatology, Saveetha Medical College and \\ Hospital, Chennai, Tamil Nadu, India
}

\begin{abstract}
Address for correspondence Hema V. Varadarajulu, DCH, DNB, DM, 18, 3rd main road, Nanganallur, Chennai 600 061, Tamil Nadu, India (e-mail: sathyahema3@yahoo.co.in).
\end{abstract}

\author{
Abstract \\ Keywords \\ - gastric antral web \\ - dyspepsia \\ - gastric outlet \\ obstruction
}

We report the case of a 65-year-old patient with rheumatoid arthritis presenting with dyspeptic symptoms. Upper gastrointestinal (UGI) endoscopy revealed a gastric antral web, which was confirmed by UGI series. The presence of webs in the gastrointestinal tract, excluding the esophagus, is a rarity. Here, we highlight the anatomical variations in antral webs and endoscopic diagnosis, which ultimately influence the type of endotherapy.

\section{Introduction}

Recent onset dyspepsia with vomiting in an elderly person is an alarming symptom and an indication for an early upper gastrointestinal (UGI) endoscopy. Here, we present the case of an elderly male with dyspeptic symptoms due to a less frequently encountered benign pathology.

\section{Case Report}

A 65-year-old male patient suffering from rheumatoid arthritis presented with a 6 -month duration of postcibal bloat and early satiety. UGI endoscopy revealed a crescentic mucosal fold narrowing the antral lumen (mimicking a pylorus), $2 \mathrm{~cm}$ before the normal pylorus ( $\boldsymbol{\sim}$ Fig. 1a). A 9.2-mm-diameter
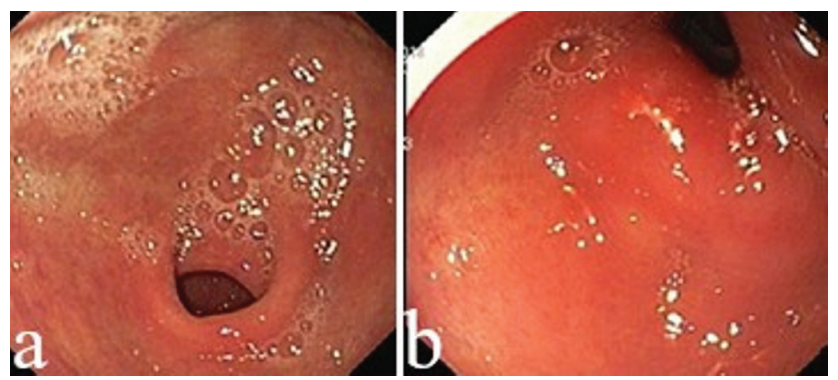

Fig. 1 (a) Upper gastrointestinal endoscopy showing a persistent, overhanging, crescentic mucosal fold narrowing the antrum and creating a pyloruslike orifice. (b) Normal pylorus. gastroscope traversed the pseudopylorus, antral tunnel, and

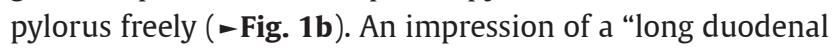
bulb" on the way in prompted a reassessment of the antropyloric and duodenal regions during withdrawal of the scope. Recognition of the antral mucosal fold creating a pseudopylorus, antral tunnel mimicking a duodenal bulb but without the corresponding mucosa, and a normal pylorus lead to the detection of gastric antral web (GAW) in a single UGI endoscopy examination. The mucosal fold was biopsied, which was later reported as Helicobacter pylori negative chronic gastritis, negative for malignancy. A Barium study performed subsequently demonstrated the incomplete antral web with minimal luminal narrowing (-Fig. 2). The patient was managed medically since the web was nonobstructive.

\section{Discussion}

GAW or antral mucosal diaphragm is a rare lesion reported in all ages, with an incidence of $0.14 \%$ in adults. ${ }^{1,2}$ It consists of a layer of mucosa and submucosa lying perpendicular to the long axis of the stomach conjectured to occur during the healing of circumferential prepyloric ulcers.

UGI endoscopy reveals a persistent circumferential membrane with a fixed-size central or eccentric aperture. The other anatomical variants include overhanging crescentic folds or circumferential redundant folds. All the three types are unaffected by peristalsis or gastric insufflation and mistaken for pylorus. ${ }^{3}$ 


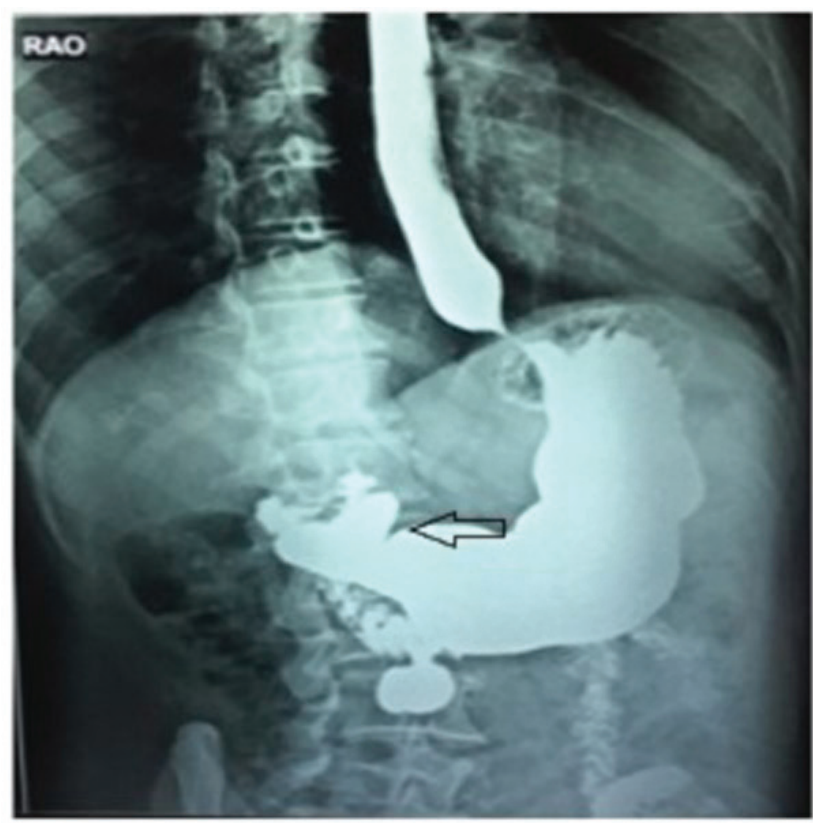

Fig. 2 Persistent, eccentric, incomplete, thin, linear, intraluminal filling defect seen in the superior border of the distal gastric antrum.

Contrast studies reveal persistent thin radiolucent line in the antrum projecting from the greater and lesser curves, and a "double-bulb" sign.

GAW with aperture greater than $1 \mathrm{~cm}$ presents with dyspeptic symptoms, usually managed with acid suppression and dietary modification. Aperture less than $1 \mathrm{~cm}$ causes gastric outlet obstruction [GOO] symptoms necessitating surgery or endotherapy: balloon dilatation for minimal luminal obstruction, radial incisions with a papillotome for partial obstruction, and resection with snaring of redundant folds in obstructive type. Though the absence of muscularis propria in GAW makes complications less likely, endotherapy should be performed cautiously. ${ }^{2}$

\section{Conclusion}

Despite the rarity, awareness of GAW helps the endoscopist to identify and manage appropriately.

\section{Funding}

None.

\section{Conflict of Interest}

None.

\section{References}

1 Gul W, Abbass K, Markert RJ, Barde CJ.. Gastric antral web in a 103-year-old patient. Case Rep Gastrointest Med 2011;2011: 957060

2 Morales SJ, Nigam N, Chalhoub WM, Abdelaziz DI, Lewis JH, Benjamin SB. Gastric antral webs in adults: a case series characterizing their clinical presentation and management in the modern endoscopic era. World J Gastrointest Endosc 2017;9(1):19-25

3 Fritz J, Martinez A, Arca M, Lerner D. Endoscopic diagnosis of antral webs in children. Endoscopy 2017;49(S 01):E18-E20 